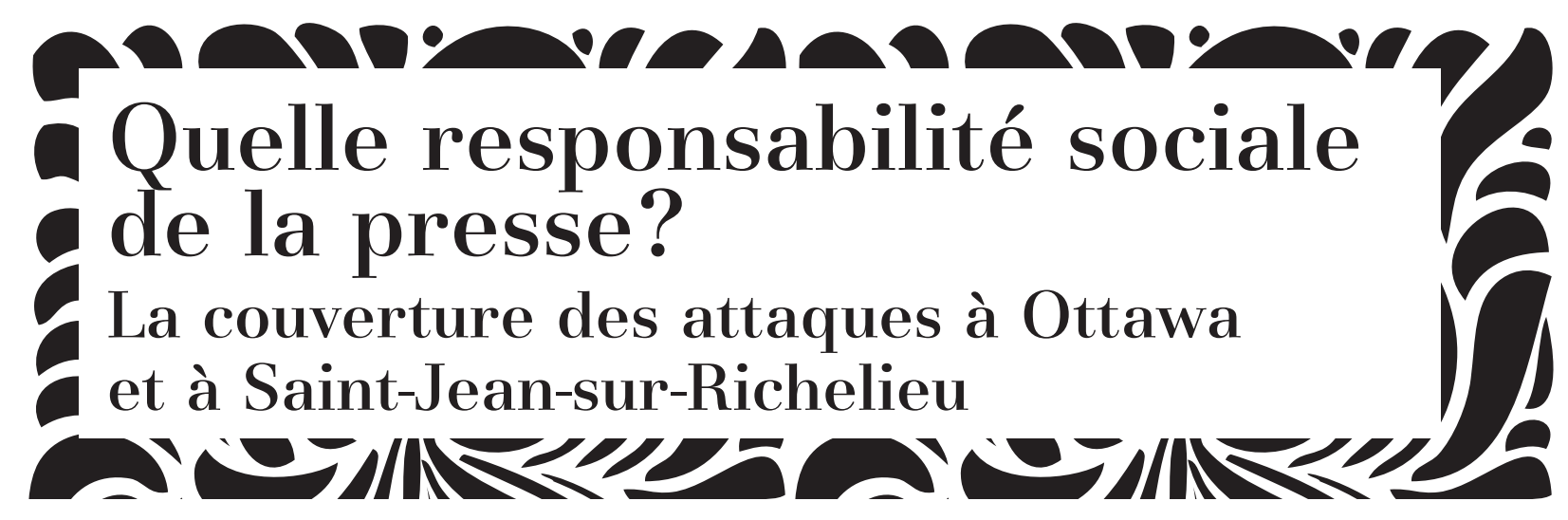

\author{
Marie-Eve Carignan \\ Professeure adjointe \\ Département des lettres et communications \\ Université de Sherbrooke \\ Canada \\ marie-eve.carignan@usherbrooke.ca
}

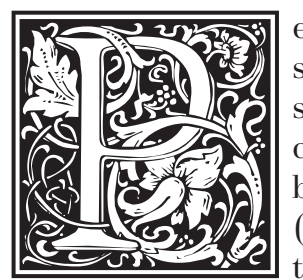

erçus d'abord comme l'expression d'un idéal démocratique et social, outil essentiel de la publicité des idées et de l'espace public, tel que décrit par Habermas (1991), les médias se sont longtemps développés conformément aux théories libérales prônant une liberté essentielle de la presse qui a trouvé écho dans nombre de chartes et de textes de loi. Ces principes de liberté et d'indépendance quasi absolus, bien que limités par l'application d'autres droits et libertés, suscitent toutefois certaines critiques qui se sont exprimées dès les recherches des années cinquante, alors que Siebert, Peterson et Schramm (1956) s'inspiraient des conclusions de la Commission on Freedom of the Press, communément appelée Commission Hutchins (1947), pour formuler la théorie de la responsabilité sociale de la presse. Cette théorie affirme que la liberté de presse s'accompagne d'importantes responsabilités afin de permettre aux médias d'assumer pleinement leur rôle au sein des sociétés démocratiques. Elle soutient que les médias doivent s'assurer d'agir de façon responsable et que la société est en droit d'exiger des comptes pour veiller à ces agissements responsables, d'où le principe d'imputabilité des médias et l'engagement à fournir au public une information juste et de qualité.

Ces concepts trouvent écho dans les codes déontologiques actuellement adoptés par les médias, les

Pour citer cet article, to quote this article, para citar este artigo :

Marie-Eve Carignan «Quelle responsabilité sociale de la presse? La couverture des attaques à Ottawa et à Saint-Jean-sur-Richelieu ", Sur le journalisme, About journalism, Sobre jornalismo [En ligne, online], Vol 7, $\mathrm{n}^{\circ} 1$ - 2018, 15 juin June 15 - 15 de junho.

URL : http://www.surlejournalisme.com/rev 
professionnels de l'information et les organismes d'autorégulation. Ce sont aussi les principes soutenus par la théorie de la responsabilité sociale de la presse qui ont conduit à la création d'organismes d'autorégulation médiatique, tel le Conseil de presse du Québec (CPQ), créé en 1973 afin de veiller " à la protection de la liberté de la presse et à la défense du droit du public à une information de qualité " (Conseil de presse du Québec, 2016, en ligne). Saint-Jean note que "la longévité appréciable et la philosophie antiformaliste " (Saint-Jean, 2002, p. 96) du CPQ figurent parmi les éléments qui ont sans doute le plus contribués à sa reconnaissance, mais que, bien que l'organisme soit généralement respecté et estimé dans les milieux journalistiques, les " critiques les plus fondamentales " qui lui sont adressées concernent son mode de fonctionnement, puisque :

"faute de moyens et à cause de la pauvreté de ses ressources, le CPQ restreint sa réflexion à l'étude des dossiers qui lui sont soumis et procède au cas par cas selon les plaintes reçues, sans effectuer de véritables enquêtes ou de recherches approfondies, qui permettraient de situer les problèmes dans le contexte plus général du monde des médias. " (Saint-Jean, 2002, p. 98)

Depuis 2010, le conseil d'administration de l'organisme et son mode de fonctionnement se retrouvent aussi grandement fragilisés par le départ du groupe médiatique Québecor ${ }^{1}$. Ces limites de fonctionnement restreignent notamment les capacités de recherche, d'autosaisie et de réflexion du $\mathrm{CPQ}$, ce qui le conduit à être réactif aux plaintes qui lui sont présentées et à peu se positionner sur les enjeux liés aux modifications des pratiques journalistiques, alors que le développement accéléré des technologies de l’information crée une pression temporelle sur les professionnels de l'information, notamment par l'usage des médias sociaux. Les principes déontologiques et les codes professionnels semblent peu s'être adaptés à cette nouvelle réalité professionnelle qui pose différents défis, particulièrement en situation critique (Carignan, 2014 ; Bettez, 2010).

En plus des critiques récurrentes qui lui sont adressées concernant ses difficultés à remplir sa mission d'autosaisie et quant au manque de diffusion, et parfois de constance, de ses décisions ${ }^{2}$, l'efficacité du CPQ est fréquemment remise en doute, alors que plusieurs chercheurs questionnent la valeur des sanctions morales, affirmant que le modèle d'autorégulation des médias s'est soldé par un échec (voir notamment le rapport de Leveson, 2012, sur la Press Complaints Commission Britannique ou les travaux de Fengler et al., 2015 ; Bernier, 2013 ; Corriveau et Sirois, 2012).

Ce contexte conduit ces chercheurs à affirmer qu'il faut revoir les modes de régulation pour permettre aux médias de remplir pleinement leurs responsabilités sociales. C'est ce qui nous pousse à nous intéresser aux limites actuelles de l'application des principes déontologiques lors de la couverture de crises. Cette analyse nous permettra de cerner les limites de la théorie de la responsabilité sociale de la presse et d'offrir des pistes de réflexion pour assurer une meilleure concordance entre les principes déontologiques et les pratiques professionnelles.

Parmi les nombreuses crises à être traitées par les médias, la couverture des attentats et des fusillades suscite, depuis plusieurs années, divers questionnements particuliers sur les pratiques journalistiques à adopter dans ce genre de situations. Ayant eux-mêmes à négocier avec ces événements troublants, mettant parfois en jeu leur propre sécurité et celle de leurs proches, dans un contexte d'instantanéité de l'information et de recherche d'exclusivité, les journalistes ont été confrontés à différents défis déontologiques et éthiques dans la couverture d'attentats récents. De nombreuses questions éthiques, entourant notamment les images qu'il convient de diffuser, les termes adéquats à utiliser ou les sources d'informations qu'il convient de mobiliser et de valider poussent les chercheurs, les gestionnaires des entreprises de presse et la communauté journalistique elle-même à se questionner sur ces pratiques. De plus, la diffusion en direct des événements sur les chaînes d'information continue et les réseaux sociaux, l'intervention spontanée du public via internet et la baisse du nombre de journalistes œuvrant au sein d'entreprises de presse de plus en plus concentrées, ajoutent au questionnement.

Ainsi, les professionnels de l'information ont interpelé le CPQ, en 2006, suivant les critiques suscitées par la diffusion d'informations erronées entourant la fusillade au Collège Dawson de Montréal, afin que l'organisme se penche sur la médiatisation des situations de crise et offre de nouveaux outils déontologiques mieux adaptés pour permettre aux médias de faire correctement leur travail dans ce type de situation. Or, en raison de moyens financiers précaires, d'un manque de ressources humaines et de divergences sur sa mission et ses priorités au sein de son conseil d'administration (ce qui ramène aux limites du CPQ précédemment évoquées par SaintJean et à la frilosité de l'organisme devant l'autosaisie), le comité-conseil mis en place par l'organisme n'a pas été en mesure de donner de suites à son rapport préliminaire. 
Ayant participé aux activités de ce comité-conseil et souhaitant poursuivre la réflexion en posant un regard sur les pratiques adoptées lors de récents événements d'actualité qui ont bouleversé les citoyens et la communauté journalistique, nous souhaitons ici analyser les pratiques professionnelles mobilisées dans le traitement médiatique des attentats, en opposant ces pratiques aux principes généralement reconnus dans les codes déontologiques de la profession journalistique. Cette recherche veut également susciter divers questionnements sur les limites actuelles de l'autorégulation des médias. Pour ce faire, nous nous intéresserons spécifiquement à la couverture des attaques survenues à l'automne 2014 au Canada.

$\mathrm{Au}$ cours des prochaines pages, nous reviendrons sur ces événements et présenterons quelques exemples de pratiques journalistiques pouvant être mises en parallèle avec les principaux griefs relevés dans la jurisprudence du CPQ et avec les principaux énoncés du Guide de déontologie journalistique du Conseil de presse du Québec (CPQ, 2015a) afin d'observer les limites potentielles d'application des principes de l'organisme. La démarche proposée ici se situe donc à cheval entre une approche empirique et une réflexion théorique sur l'application des principes d'autorégulation lors de la couverture d'attentats.

\section{LES ÉVÉNEMENTS SURVENUS}

Les années 2014 à 2016 furent particulièrement chargées, notamment en Europe et au Canada, en matière d'attaques armées perpétrées contre de forts symboles sociaux. Ces attaques, principalement revendiquées par des individus disant agir au nom de l'islam et du groupe armé État Islamique $(\text { ÉI })^{3}$, ont particulièrement ébranlé la société occidentale puisqu'elles ont démontré sa vulnérabilité devant ce type de menaces qui ont pris pour cibles des institutions sociales hautement symboliques, que ce soit les institutions politiques, militaires ou médiatiques, attaquant alors des principes fondateurs des sociétés démocratiques, telle la liberté d'expression qui leur est associée, en plus de susciter de nombreux questionnements devant le départ et la radicalisation de citoyens désirant aller « faire le djihad ». Ces attaques, qualifiées par certains médias et chercheurs d'actes terroristes ${ }^{4}$, ont été perpétrées tant par des groupes organisés que par des individus isolés, souvent définis comme des loups solitaires, traduction du terme lone wolf qui réfère à :

"[...] a person who acts on his or her own without orders from - or even connections to - an organization. [...]
A lone wolf is a standalone operative who by his very nature is embedded in the targeted society and is capable of self-activation at any time. [...]

Some lone wolves are ideologically motivated, some are religiously inspired, some are mentally disturbed and still others are influenced by a combination of these factors. [...] We distinguish between lone wolves and «lone nuts» because, although many politically motivated attackers do have some degree of mental illness, rational and irrational individuals operate differently. Mentally disturbed individuals are far more likely to self-radicalize in a vacuum and have less concern for their own safety than do most politically motivated attackers. This lack of concern for their own safety often helps them to overcome their lack of skill. " (Burton et Stewart, 2008, paragr. 7-12)

Les principales caractéristiques des actions «terroristes " des loups solitaires (lone wolf terrorists), selon Spaaij (2012), sont qu'ils opèrent individuellement, n’appartiennent pas à un réseau ou à un groupe terroriste organisé et que le modus operandi est conçu et exécuté par l'individu sans commande directe d'une autorité hiérarchique, ce qui semblait correspondre aux profils des auteurs des attaques étudiées aux fins de la présente analyse de cas, soit celles survenues à l'automne 2014 au Canada.

Ces dernières remontent d'abord au 20 octobre 2014, dans un stationnement commercial de SaintJean-sur-Richelieu, au Québec, alors qu'une voiture a foncé sur deux militaires de l'Armée canadienne. Un " adepte de l'islam radical » (Société Radio-Canada, 9 décembre 2014), Martin « Amad " Couture-Rouleau, 25 ans, a alors causé la mort de l'adjudant Patrice Vincent et blessé un second militaire, en fonçant sur ces derniers en voiture, avant d'être lui-même abattu par les policiers. Il avait précédemment contacté les services d'urgence en précisant avoir agi " au nom d'Allah " (Deland, 20 octobre 2014). Actif sur les réseaux sociaux où il faisait " de la propagande djihadiste depuis des mois " (La Presse, 20 octobre 2014), l'homme était connu des autorités policières provinciales et fédérales.

Deux jours après ces événements, le 22 octobre 2014, deux fusillades sont survenues sur la colline parlementaire d'Ottawa, en Ontario. Michael Zehaf-Bibeau, également connu des autorités policières et décrit comme un "désillusionné nourrissant des croyances extrémistes " (Société Radio-Canada, 23 octobre 2014), a fait feu devant le Monument commémoratif de guerre puis à l'hôtel du parlement canadien, causant la mort du caporal Nathan Cirillo, 
avant d'être abattu par le sergent d'armes Kevin Vickers, ancien officier de la Gendarmerie royale du Canada (GRC), alors chef de la sécurité du Parlement. L'attaque aura duré " moins de cinq minutes » selon la GRC (Le Monde et AFP, 23 octobre 2014).

Zehaf-Bibeau et Couture-Rouleau ont en commun d'avoir agi seuls. Dans une vidéo rendue publique par la GRC en mars 2015, Zehaf-Bibeau affirme avoir commis ces attentats " pour venger les moudjahidines du monde entier ", car il estime que le Canada serait " devenu officiellement un de nos ennemis en nous déclarant la guerre et en nous bombardant ainsi, créant beaucoup de terreur dans nos pays en nous assassinant et tuant nos innocents " (Société Radio-Canada, 6 mars 2015). Il affirme avoir ciblé des soldats pour montrer que personne ne serait en sécurité au Canada.

Rapidement, en réaction à ces événements, les bases militaires canadiennes ont renforcé leurs mesures de sécurité et ont été fermées aux visiteurs. De plus, le commandant de la $2^{\mathrm{e}}$ Division du Canada a transmis une directive particulièrement rare à tous les militaires les informant que, jusqu'à nouvel ordre, ils ne devaient pas être dans des lieux publics en uniforme afin d'assurer leur sécurité (Ricard-Châtelain, 22 octobre 2014). Cette mesure a alimenté plusieurs discussions sur l'équilibre à trouver entre l'importance d'assurer la sécurité des militaires, de protéger l'image de l'armée et de se montrer intransigeant face au terrorisme et aux attaques.

Ces deux attaques rapprochées ont suscité un intérêt médiatique international important. Bien que divers médias internationaux aient souligné la qualité de la couverture réalisée par les médias canadiens en direct lors des événements d'Ottawa, notamment en raison du calme et du professionnalisme du présentateur de The Canadian Broadcasting Corporation $(C B C)$, Peter Mansbridge, et aient estimé que ces médias auraient pu donner une leçon aux médias américains et français (Collard, 24 octobre 2014 ; West, 22 octobre 2014), certains aspects de la couverture méritent une réflexion sur la responsabilité de la presse et une mise en parallèle avec quelques grands principes issus du guide de déontologie du CPQ et de la jurisprudence de l'organisme. L'attaque d'Ottawa sera rapidement qualifiée, par divers médias et intervenants politiques, comme un acte terroriste et largement attribuée aux activités de l'ÉI. La couverture de cet événement en direct, la mise en parallèle avec celui de Saint-Jean-sur-Richelieu, la reprise par les médias traditionnels des interventions sur les médias sociaux, dont celles du tireur lui-même, ainsi que la retransmission en direct d'un discours à la nation émotif et largement axé sur la résistance face au terrorisme, réalisé par l'ancien premier ministre canadien Stephen Harper, nous poussent à nous intéresser à la médiatisation de ces événements comme point de départ de la présente analyse.

\section{APPROCHE THÉORIQUE ET PROBLÉMATIQUE}

\section{La perception du terrorisme dans la société du risque médiatisé}

Giddens (2000, 2005), Beck (2001) et PerettiWatel (2001) décrivent la société contemporaine comme une société du risque, où les développements technologiques et industriels nous confrontent à des dangers d'une nouvelle nature, causés par l'Homme, incontrôlables et non limités géographiquement. Lagadec parle alors d'une " dislocation de nos environnements et socles de référence " (Lagadec, 2008, p. 13). Quatre risques majeurs seraient typiques de cette société : les catastrophes écologiques, l'effondrement des mécanismes de la croissance économique, le développement du totalitarisme et les conflits de grande ampleur (Giddens, 2000). La crainte de voir ces risques se potentialiser crée une " culture du risque » qui fait notamment craindre les conflits, les attentats et les actes qualifiés de terroristes, devenant ainsi un important argument économique (pensons à la vente d'assurances, de médicaments, d'armements), en plus de modifier les formes de lien social (Beck, 2001). Les risques ont profondément pénétré le quotidien des citoyens, puisque les potentialités de voir ces risques se concrétiser ne cessent de croître, dont celles liées aux menaces terroristes (Centers for Disease Control and Prevention, 2012). Le Centers for Disease Control and Prevention (CDC) estime que les terroristes parviennent efficacement à déjouer le contre-terrorisme en raison notamment de leurs habiletés technologiques et de leur stratégie consistant à viser des cibles vulnérables ou à provoquer plusieurs attaques simultanément afin de tuer ou de blesser de nombreuses personnes à la fois. Nacos (2005) aborde aussi cette stratégie qui consiste à créer des événements de grande ampleur et à faire le plus de victimes possible pour attirer l'attention des médias, de la population et des gouvernements non seulement des pays pris pour cibles, mais du monde entier. Elle nomme cette stratégie «terrorisme à finalité médiatique » (mass-mediated terrorism).

La communication des risques, définit comme la capacité d'informer une audience sur le type et la magnitude des conséquences possibles, résultant d'un comportement ou d'une exposition au risque, ainsi que sur les probabilités d'occurrences (CDC, 2012), est grandement limitée par la pratique médiatique qui consiste à rapporter des événements 
concrets et visibles, d'où l'intérêt des terroristes à provoquer des événements de grande ampleur pour attirer l'attention médiatique. Dans une société axée sur la « culture du visible » et du matériel, un mal invisible ne pourra rivaliser avec un bien concret. Ainsi, les médias chercheront toujours à rapporter des situations perceptibles pour le public, qui répondent aux critères de définition d'une nouvelle. Ce besoin de tangibilité pourra supprimer la perception des risques, mais pas leur réalité ni leurs effets. Cette place prépondérante accordée au matériel constituera alors un terreau culturel et politique idéal pour la prolifération des risques, les risques niés étant ceux qui prolifèreront le plus vite (Beck, 2001).

Raboy soulève ainsi que les médias doivent attendre " qu'une mise en situation soit provoquée par un événement spectaculaire » (Raboy, 1993, p. 102) avant de pouvoir s'intéresser aux problèmes sociaux et aux crises qui affectent la société, en plus d'être restreints par l'espace ou le temps d'antenne disponible ainsi que par la structure des médias qui limite leur capacité « de contextualiser l'information sociale et d'en expliquer le sens " (Raboy, 1993, p. 84, basé sur Tuchman, 1978, Carey, 1986 et Miège et al., 1986). Néanmoins, la perception des risques serait affectée par leur médiatisation en raison de l'importance de la couverture des catastrophes et de l'internationalisation des informations, rendue possible par les nouvelles technologies, qui créerait un " rapprochement médiatique » et donnerait au public la perception que les risques mêmes lointains les guettent directement (Boutté, 2006). Le concept de "société du risque " serait même indissociable des médias, puisque parler des risques serait presque toujours parler de leur médiatisation (Champagne, 1999). En ce sens, les études sur la perception du risque (Fischhoff et al., 1978) démontrent que les publics surestiment les « risques imaginaires " ou la fréquence des événements spectaculaires et fortement médiatisés (attentats, meurtres, suicides, etc.), pourtant moins fréquents, et sous-estiment les risques naturels qui sont les plus fréquents, mais les moins médiatisés (tels les maladies et les accidents). La façon dont les médias traitent un sujet jouerait donc un rôle clé dans la perception du risque chez les individus qui évalueraient les événements par une comparaison à ceux qui ont été fortement médiatisés préalablement (Boutté, 2006). Une attaque revendiquée par un groupe armé sera ainsi constamment comparée aux précédents événements similaires.

Les médias auront également tendance à donner un nom aux événements d'envergure qu'ils sont appelés à couvrir, pensons par exemple au «9/11» ou, plus récemment, à " l'attaque contre Charlie Hebdo ». Or, le fait de nommer ou d'attribuer une qua- lification particulière à un événement pourrait jouer sur la compréhension des publics de son importance. Le choix des termes devient donc particulièrement déterminant dans la couverture des événements critiques. En revenant sur la médiatisation de la fusillade survenue le 6 décembre 1989 à l'École polytechnique de Montréal, au Québec, Raboy, en se basant sur Pêcheux (1988), évoquait déjà une " lutte discursive - une bousculade en vue de nommer les choses d'une certaine manière, dans le sillage de l'événement " (Raboy, 1993, p. 83), afin de le contextualiser et d'en interpréter le sens. Ainsi, les termes utilisés pour décrire les fusillades et les attaques revendiquées par des sympathisants de groupes extrémistes suscitent une vive réflexion sur les impacts de choix discursifs. Pensons, par exemple, au vocable associé au terrorisme qui est souvent contesté et est loin de faire l'unanimité dans la communauté scientifique et politique (Spaaij, 2012).

Ce contexte nous amène donc à interroger le rôle des médias dans la couverture des attentats. La façon de couvrir ce type d'événements est de nature à encourager une réflexion éthique et, éventuellement, à pousser les organismes d'autorégulation à revoir les règles déontologiques en place. C'est ce qui nous conduit à interroger l'efficacité des outils déontologiques actuellement mis à la disposition des professionnels de l'information pour les aider à réaliser ce type de couverture. Nous nous penchons ici spécifiquement sur la couverture des attaques d'octobre 2014 au Canada pour observer si certaines pratiques des médias canadiens posent des questionnements quant au respect des principes présentés dans le guide déontologique et au sein de la jurisprudence du CPQ et soulèvent les limites de la responsabilité sociale des médias lors de ce type de couverture.

\section{Méthodologie}

L'importance des risques en société, dont ceux liés aux fusillades et aux attentats, de même que le rôle accordé aux médias dans la communication de ces risques, nous incitent donc à analyser plus spécifiquement les pratiques journalistiques lors de la couverture des attentats. Comme les attaques revendiquées par des individus isolés et des groupes organisés ont largement occupé l'actualité internationale ces dernières années, suscitant une certaine crainte chez les publics et une interrogation sur les enjeux liés à la radicalisation, et puisque les professionnels de l'information ont eux-mêmes interpelé les instances d'autorégulation afin d'être mieux outillés pour traiter ces situations, nous croyons qu'il est nécessaire d'analyser la couverture de ce type d'événement afin de la mettre en parallèle avec les principes déontologiques reconnus par la profession. 
Partant de ces constats généraux, nous avons décidé de nous intéresser plus spécifiquement aux attaques survenues en octobre 2014 au Canada, afin d'établir une relation entre la couverture de ces événements par les médias au Québec et le respect des principes déontologiques reconnus par la profession journalistique. Nous avons ainsi relevé les principes du guide de déontologie du CPQ (2015a) pouvant s'appliquer directement au traitement d'attaques armées ou d'événements critiques et les avons confrontés à différentes pratiques professionnelles observées lors d'une analyse de la couverture médiatique des attaques de 2014, ce qui nous a permis de soulever divers questionnements sur l'application de ces principes.

Notre corpus était constitué des exemplaires imprimés des journaux québécois Le Devoir, La Presse, The Gazette, Le Soleil, Le Nouvelliste, La Tribune, La Voix de l'Est et Le Droit, publiés entre le 20 octobre 2014, date des événements de SaintJean-sur-Richelieu, et le 27 octobre 2014, cinq jours après ceux d'Ottawa. Nous avons ajouté à ce corpus une revue de presse effectuée en ligne sur les sites de Radio-Canada, de TVA Nouvelles, du Journal de Montréal et du Journal de Québec. Notre objectif était d'identifier si des manquements potentiels pouvaient être observés et des questionnements éthiques pouvaient être soulevés en regard des pratiques professionnelles adoptées pour couvrir ces événements. De ce fait, nous n'avons pas effectué une analyse exhaustive du corpus, mais avons plutôt effectué une lecture complète de celui-ci dans le but d'isoler, dans un premier temps, des exemples d'articles qui nous semblaient soulever des questionnements éthiques quant aux principes invoqués dans le guide du CPQ et dans la jurisprudence de l'organisme. Cet exercice nous a fourni suffisamment d'exemples, sur chacun des principes, pour étoffer cette analyse de cas et nourrir notre questionnement. Il serait toutefois intéressant, dans un deuxième temps, d'effectuer une analyse plus approfondie de ce corpus.

Nous nous sommes ainsi particulièrement intéressés aux sections $\mathrm{C}$ "Recherche de la vérité » et $\mathrm{D}$ «Respect des personnes et des groupes » du guide du CPQ (2015a) qui s'appliquaient le plus directement au cas à l'étude. D'ailleurs, le Rapport d'activités 2014 - 2015 du CPQ (2015b) souligne que les principaux griefs invoqués dans les plaintes étudiées par le tribunal d'honneur de l'organisme, lors de sa dernière année d'exercice, en regard de manquements potentiels à la déontologie journalistique dans la presse écrite ou électronique, concernent principalement ces sections, une tendance qui se confirme d'une année à l'autre dans les rapports d'activités de l'organisme. En effet, "la majorité des motifs invoqués concernent la qualité de l'information " (CPQ, 2015b, p. 19), soit 53\% des motifs de plaintes étudiés. Vient ensuite "l'attitude des médias à l'égard des personnes et des groupes, dans une proportion de 24\% " (CPQ, 2015b, p. 19). Concernant la qualité de l'information, les principaux griefs relevés concernent les informations incomplètes ou inexactes, le manque d'équilibre de l'information, la partialité ainsi que la publication de photographies, de titres, de manchettes ou de légendes et le sensationnalisme. En ce qui concerne l'attitude des médias à l'égard des personnes et des groupes, les principaux griefs relevés concernent les préjugés, l'atteinte au droit à un procès juste et équitable, l'atteinte au droit à la vie privée, les propos méprisants, les propos haineux et racistes, les propos islamophobes et l'atteinte au droit à la dignité humaine.

Nous mettrons donc en parallèle les principes invoqués dans le Guide de déontologie journalistique du Conseil de presse du Québec (2015a) ainsi que les principaux griefs issus de sa jurisprudence avec certaines pratiques observées dans les médias pour traiter des événements de Saint-Jean-sur-Richelieu et d'Ottawa. Cette analyse permettra d'entamer une réflexion sur la viabilité de la théorie de la responsabilité sociale de la presse et des processus d'autorégulation médiatiques actuellement en place ainsi que sur le rapport entre liberté de presse et droits individuels (vie privée, sécurité, respect des minorités) ainsi qu'entre intérêt public et curiosité publique.

\section{RECHERCHE DE LA VÉRITÉ ET QUALITÉ DE L'INFORMATION}

Concernant l'exactitude et la qualité de l'information, cette analyse nous permet d'abord de soulever une réflexion sur le recours aux qualificatifs liés au terrorisme dans les médias, dans un contexte où l'activité terroriste est, comme nous l'évoquions précédemment, l’objet de nombreuses définitions et interprétations et où aucun consensus ne semble se dessiner au sein des communautés scientifiques ou politiques. Il convient donc de réfléchir aux implications de ces choix terminologiques.

À la suite des événements d'octobre 2014, un débat a rapidement surgi dans différents médias canadiens qui interrogeaient les liens entre les auteurs des attaques et les mouvements terroristes. Certains médias ont rapidement parlé d'actes terroristes organisés adressés contre l’Armée canadienne ou contre le Canada tout entier, alors que d'autres ont évoqué des attaques isolées causées par la maladie mentale ou ont qualifié les auteurs de loups solitaires, référant ici notamment aux caractéristiques du lone wolf terrorist évoquées plus haut. Ces dif- 
férences discursives donnent un sens fort varié aux événements et à leur contexte. De plus, certains médias canadiens et intervenants politiques ont rapidement effectué des liens entre les responsables de ces attaques et les mouvements terroristes (principalement l'ÉI), sans que la GRC se soit encore prononcée à ce propos.

Un second aspect de la couverture médiatique relative à ces événements et à leur contextualisation qui retient l'attention est le fait que plusieurs médias aient rapidement amalgamé les événements de SaintJean-sur-Richelieu et ceux d'Ottawa, en raison des dates rapprochées et de certaines similarités chez les auteurs des attaques qui, notamment, s'étaient tous deux préalablement radicalisés et s'en sont pris à des représentants des institutions miliaires canadiennes. Ce rapprochement a pu augmenter la crainte des publics et leur donner l'impression d'avoir affaire à des actes terroristes organisés, ce qui rejoint l'impact potentiel de la médiatisation sur la perception des risques évoqué plus tôt. Des médias ont ainsi juxtaposé les photos de Zehaf-Bibeau et de Couture-Rouleau pour illustrer les événements (on en retrouve un bon exemple dans le Vancouver Observer du 24 octobre 2014 ou dans l'article de Gollom du 27 octobre 2014 sur le site de $C B C$ News), alors que la GRC a affirmé qu'elle n'avait pas d'information lui permettant d'établir un lien entre les deux attaques.

Le Guide de déontologie journalistique du Conseil de presse du Québec précise, au sujet de la présentation de l'information, que la déontologie veut que les "journalistes et les médias d'information respectent l'intégrité et l'exactitude de l'information dans la présentation et l'illustration qu'ils en font " (CPQ, 2015a, p. 21). De plus, au sujet du sensationnalisme, il est prévu que les "journalistes et les médias d'information ne déforment pas la réalité, en exagérant ou en interprétant abusivement la portée réelle des faits et des événements qu’ils rapportent " (CPQ, 2015a, p. 21).

Or, dans un contexte où le terrorisme est très difficile à définir et à circonscrire et, dans le cas d'événements où les gestes ont été commis par des individus agissants seuls, il est particulièrement difficile de déterminer où commence et où s'arrête l'acte terroriste. Au Canada, le Code criminel, à l'article 83.01, définit l'« activité terroriste " comme étant un acte commis " au nom exclusivement ou non - d'un but, d'un objectif ou d'une cause de nature politique, religieuse ou idéologique » en vue de susciter la crainte chez la population « quant à sa sécurité, entre autres sur le plan économique, ou de contraindre une personne, un gouvernement ou une organisation nationale ou internationale à accomplir un acte ou à s'en abstenir » (extrait du Code criminel canadien, 1985). En ce sens, les actes analysés peuvent se rapprocher de la définition canadienne du terrorisme, puisqu'ils visaient à susciter la crainte, même lorsque posés par des individus agissants seuls.

En dépit des questionnements et des différentes positions, parfois contradictoires, qui ont été exprimées dans les médias quant à la nature des gestes commis à Saint-Jean-sur-Richelieu et à Ottawa, nous pouvons souligner la place que les médias canadiens ont accordée aux différentes opinions afin de permettre aux intervenants et aux publics de réfléchir au sens donné à ces événements et de déterminer s'ils répondaient ou non à la définition d'« actes terroristes ». Dans le contexte où des élections fédérales étaient imminentes au Canada, que la sécurité nationale risquait d'y être un enjeu de taille et que le gouvernement fédéral conservateur de l'époque a rapidement présenté les événements d'octobre 2014 comme des actes terroristes ${ }^{5}$, justifiant du même coup l'adoption du projet de loi antiterroriste C-51 permettant d'accroître le pouvoir des forces de sécurité, ce débat était d'autant plus important pour permettre aux publics de réfléchir sur les enjeux liés à la sécurité publique et invitait les médias à une prudence singulière.

\section{De l'importance accordée aux responsables des attentats et à leur image}

Concernant l'équilibre de l'information, nous nous intéressons aussi à l'ampleur globale de la couverture de presse accordée à ces événements, en revenant sur le principe voulant que les terroristes se servent des médias pour faire entendre leurs messages (Nacos, 2005). La définition du terrorisme présentée dans le Code criminel canadien se rapproche de la vision du terrorisme à finalité médiatique développée par Nacos qui estime que les terroristes cherchent à susciter la crainte chez la population et à faire réagir le gouvernement pour ainsi donner une voix à la cause qu'ils revendiquent et qui n'est pas traitée habituellement par les médias et les politiques. De ce fait, Nacos, comme Tsfati et Weimann (2002), qualifie l'acte terroriste d'acte de communication politique. Elle estime que ce que les terroristes attendent " c'est que, confrontés à la violence politique (et plus particulièrement à des actions terroristes spectaculaires), les médias laissent passer toutes les informations relatant les faits liés à ces événements - y compris les messages savamment calculés que les terroristes veulent diffuser, qu'ils revendiquent ou non la responsabilité des actes en question " (Nacos, 2005, p. 14). Dans le cas présent, nous soulevons justement la question à savoir si les médias canadiens ont pu embarquer dans 
une stratégie savamment orchestrée et se faire les porte-parole d'idéologies prônées par les auteurs de ces attentats ou par le groupe armé ÉI qui cherche à s'y associer. Dans cet esprit, le précédent guide déontologique du CPQ, Droits et responsabilités de la presse, cité abondamment dans la jurisprudence de l'organisme, précise :

"Il est aussi de la responsabilité des entreprises de presse et des journalistes de se montrer prudents et attentifs aux tentatives de manipulation de l'information. Ils doivent faire preuve d'une extrême vigilance pour éviter de devenir, même à leur insu, les complices de personnes, de groupes ou d'instances qui ont intérêt à les exploiter pour imposer leurs idées ou encore pour orienter et influencer l'information au service de leurs intérêts propres, au détriment d'une information complète et impartiale. " (Conseil de presse du Québec, 2003, p. 16)

Il est donc légitime de se demander si les terroristes et les auteurs d'attaques cherchent à utiliser les médias pour obtenir une certaine gloire, en plus d'en faire une caisse de résonnance de leurs messages et revendications. Les procédés utilisés par l'ÉI sur le web et les médias sociaux vont notamment en ce sens. Plusieurs chercheurs soulèvent les dangers potentiels d'accorder trop de place et d'importance aux responsables d'événements violents et de diffuser leur propagande (Hénin, 2015 ; Berthomet, 2015 ; Carignan, 2014 ; Brillon, 2007 ; Nacos, 2005), ce qui pourrait notamment avoir pour effet de les glorifier et d'inciter des imitateurs, individus possiblement dérangés à la recherche d'une certaine glorification, à reproduire des actes de violence. Estimant, en ce sens, que la diffusion des images ou des vidéos préparées par des individus radicalisés pouvait " contribuer au terrorisme " en "transformant les porteurs du message en "vedettes" ", la Sûreté du Québec a d'ailleurs invité les médias à "faire un examen de conscience " et à la prudence à la suite de la diffusion, en décembre 2014, d'une vidéo présentant « un djihadiste canadien proférant des menaces contre le Canada au nom du groupe armé État islamique » (Radio-Canada et La Presse Canadienne, 8 décembre 2014).

Ces questionnements ne sont pas nouveaux. Ils avaient notamment nourri l'intérêt des chercheurs et des journalistes après qu'une couverture importante ait été accordée par les médias à Kimveer Gill, auteur de la fusillade au Collège Dawson de Montréal en septembre 2006, ainsi qu'à Cho Seung-hui, responsable de la fusillade de l'Université Virginia Tech aux États-Unis en avril 2007, ce dernier ayant même préparé un kit à l’intention des médias, qu’il avait posté avant la fusillade, pour s'assurer d'obtenir une couverture médiatique. Le sujet avait notamment été abordé lors d'un colloque, tenu en 2007 à la Maison de Radio-Canada et réunissant journalistes et chercheurs. Cette question a de nouveau été soulevée en juin 2014, en raison de l'attention accordée dans les médias, au Canada et à l'international, à Justin Bourque, auteur d'une fusillade qui a tué trois agents de la GRC à Moncton au Nouveau-Brunswick. Une image de Bourque en cavale lors de l'opération policière de juin 2014, vêtu d'une tenue aux couleurs militaires et d'un bandeau rappelant les héros de films d'action, a rapidement fait le tour du monde. Dietz soulevait alors la responsabilité des médias lorsqu'ils diffusent les images d'un tueur (Dietz en entrevue avec Lagacé, 2014). Revenant sur l'image de Bourque, il évoquait le risque d'inciter les imitateurs potentiels et interrogeait la pertinence de diffuser les images du tireur à l'international. Il mentionnait en particulier les dangers que pouvait provoquer la diffusion de l'image du tueur qui ressemblait aux héros de films violents et risquait d'être idolâtré par certains individus dérangés.

Le questionnement lié à la diffusion de l'image des victimes et des auteurs d'attaques était toujours d'actualité à l'automne 2014, avant les événements d'octobre, en raison de la diffusion des vidéos de décapitation de l'ÉI. Certains médias internationaux ayant parfois censuré ces images tournées par l'ÉI, d'autres les ayant diffusées intégralement, alors que certains ont préféré ne pas montrer d'images issues de l'ÉI et ont plutôt présenté des photos des victimes prises avant les faits. Le psychanalyste Vincent Magos estimait d'ailleurs que le public devrait « refuser de regarder les images des mises à mort ", non seulement pour une question de "dignité des victimes" ou pour éviter de " jouer le jeu des djihadistes ", mais aussi parce qu'elles ne laisseraient psychologiquement pas le choix au public que de «s'identifier à l'un des deux protagonistes : la victime ou son bourreau " (Magos, 15 septembre 2014).

La section 18.2 du Guide de déontologie journalistique du Conseil de presse du Québec concernant la « sensibilité du public » peut s'appliquer à ces images spécifiques :

"Les journalistes et les médias d'information évitent de diffuser inutilement des images ou propos pouvant heurter la sensibilité du public.

Lorsque le format le permet, les journalistes et les médias d'information avertissent le public que des images ou des propos choquants seront diffusés » (CPQ, 2015a, p. 26) 
Dans une décision rendue en octobre 2004, le CPQ s'est penché sur une plainte mettant en cause le Groupe TVA-LCN concernant la diffusion de la décapitation de l'américain Nick Berg. Le CPQ n’avait alors pas retenu la plainte, considérant que :

" les images diffusées au sein du reportage ne sont pas celles de la décapitation. Le choix de ne pas diffuser cette partie démontre un souci de qualité de l'information et du respect du public des médias audiovisuels du Québec. $L C N$ ne présente pas l'extrait dans son intégralité, il se termine à la limite de l'acte par le cri de la victime. Le lecteur de nouvelles de $L C N$, en prévenant les téléspectateurs du caractère violent du reportage ainsi que de la fin sonore du document, met en garde les personnes sensibles. Le Conseil rappelle que le téléspectateur est à même de juger de sa sensibilité au regard de cette mise en garde." (CPQ, 2004, D2004-05-059)

Le CPQ estimait toutefois que de diffuser ces images dans leur intégralité " aurait été un manquement à la pondération de l'information. Le fait de le[s] diffuser en partie ne peut être contestable qu'au regard de la sensibilité individuelle étant donné les avertissements liés à ces images " (CPQ, 2004, D2004-05-059). Ainsi, dans les cas de 2014, la diffusion parfois complète des images de décapitation semble alors déroger aux principes reconnus dans la jurisprudence de l'organisme.

Dans le cas des attaques d'octobre 2014, une grande importance a, encore une fois, été accordée aux responsables des événements. Les médias ayant même visité l'école secondaire où étudiait ZehafBibeau à Laval au Québec, près de vingt ans avant les événements. Différents médias, dont l'hebdomadaire Courrier Laval du 23 octobre 2014 et la chaîne d'information $T V A$, ont publié ou diffusé des images de l'école, le portrait de Zehaf-Bideau présenté dans son album des finissants ${ }^{6}$ et des photos de classe. Certains médias ont également diffusé des images d'une vidéo, rendue publique par le commissaire de la GRC en mars 2015 et tournée par Zehaf-Bibeau dans sa voiture quelques instants avant l'attaque d'Ottawa, où il évoque en partie les motivations qui le poussent à commettre ses actes violents.

Les comptes créés par Couture-Rouleau et ZehafBibeau sur divers médias sociaux ont également été scrutés à la loupe par les journalistes et ont ainsi pu servir d'équivalent au kit médias évoqué plus tôt, utilisé lors de précédentes fusillades. Ils ont permis aux médias de relayer diverses images des auteurs des attentats et de revenir sur les messages extrémistes que ces derniers diffusaient sur les réseaux sociaux notamment pour convertir de nouveaux adeptes. De plus, des photos de Zehaf-Bideau armé ont été diffusées sur des comptes Twitter liés à l'ÉI avant d'être reprises dans les médias traditionnels. Les médias ont également consacré divers articles et reportages au contenu des comptes de CoutureRouleau sur Twitter et sur Facebook, dont une page qu'il tenait sous le nom d'Ahmad LeConverti et une autre sous le nom d'Ahmad Rouleau. Des journaux, dont le quotidien La Presse, ont ainsi rapporté que Couture-Rouleau avait tenté de quitter le Canada, en juillet 2014, pour aller au Pakistan rejoindre son " meilleur ami Facebook », un résident de Karachi, avant de se faire intercepter par la police (Sioui, 24 octobre 2014) et qu'il avait longtemps été un bon vivant publiant par exemple, sur sa page Facebook, des photos de lui avec ses amis partageant une bière dans une piscine, avant de se convertir et de se mettre à véhiculer sur ses pages des théories du complot et des propos liés à l'islam radical (Peritz, Thanh Ha et Pearreaux, 21 octobre 2014). De plus, une capture d'écran du compte Facebook de Couture-Rouleau a largement circulé dans les médias (dont le site Internet de la Société Radio-Canada, 21 octobre 2014). Cette image illustre les dernières publications sur la page, parues le 17 octobre 2014, représentant deux portes, l'une menant vers l'enfer et l'autre vers le paradis.

Ainsi, les différentes photos de ces comptes ont largement nourri les médias traditionnels. Devant ces constats, la question posée dans le code du $\mathrm{CPQ}$, à savoir si les médias, même à leur insu, ne se font pas les complices de ces personnes ou de ces groupes terroristes en relayant les images et les propos qu'ils souhaitent eux-mêmes véhiculer sur diverses plateformes ou qu'ils souhaitent partager comme legs à la suite de leurs gestes semble particulièrement se poser, tout comme celle à savoir si nous n'accordons pas trop d'attention et d'intérêt aux auteurs de ces gestes. Par ailleurs, les médias sociaux facilitent l'accès à ce genre d'information, ce qui nous amène à souligner que des balises déontologiques concernant spécifiquement l'usage des informations contenues sur les médias sociaux par les journalistes pourraient les aider à évaluer davantage la pertinence de cette couverture.

\section{RESPECT DES MÉDIAS À L’ÉGARD DES PERSONNES ET DES GROUPES}

Nous avons également soulevé les principes du guide de déontologie du CPQ concernant le respect du droit à la vie privée ainsi que le respect à l'égard des personnes et des groupes, pour les analyser pa- 
rallèlement à la couverture des attaques d'octobre 2014. Le CPQ reconnait occasionnellement la pertinence de divulguer des informations relevant de la vie privée et pouvant heurter la dignité d'une personne, en se basant sur le critère d'intérêt public de l'information. Ainsi, l'article 18 du Guide de déontologie journalistique du Conseil de presse du Québec soutient que, bien que :

"Les journalistes et les médias d’information respectent le droit fondamental de toute personne à sa vie privée et à sa dignité.

Les journalistes et les médias d’information peuvent privilégier le droit du public à l'information lorsque des éléments de la vie privée ou portant atteinte à la dignité d'une personne sont d'intérêt public. " (Conseil de presse du Québec, 2015a, p. 26).

Or, la définition même d'intérêt public est largement débattue et n'est certes pas explicite. Les outils déontologiques du CPQ ne fournissent pas de définition précise de ce principe qui demeure vague et se confond souvent avec la curiosité publique. Sans définir ces deux notions, le CPQ semble tracer une ligne importante entre ce qui relève de l'intérêt public, de ce qui tient davantage de la curiosité. Sa jurisprudence rappelle que :

"Que ce soit lors de la collecte, du traitement ou de la diffusion de l'information, les médias et les journalistes doivent faire preuve de prudence, de discernement et de circonspection. Ils doivent se soucier d'informer réellement le public, et doivent faire les distinctions qui s'imposent entre ce qui est d'intérêt public et ce qui relève de la curiosité publique. [...]

Les journalistes doivent manifester à l'endroit des victimes et de leurs proches tout le respect et la compassion qui leur sont dûs (sic) en écartant les détails qui ne sont pas d'intérêt public et qui, souvent, n'ont rien à voir avec l'incident rapporté. Ces détails, davantage destinés à piquer la curiosité et qui tiennent plutôt de la surenchère, peuvent être préjudiciables à la victime ou à ses proches en les exposant à des tracas ou à des peines inutiles. Les médias et les journalistes doivent donc prendre les plus grandes précautions pour ne pas exploiter le malheur d'autrui.

Dans ce contexte, la publication de photos ou d'images et d'informations permettant l'identification des victimes ou de leurs proches doit être l'objet d'une décision basée non pas sur un simple consentement souvent obtenu sous le coup d'une émotion vive, mais d'abord et avant tout sur leur caractère d'intérêt public.

La question de l'identification des personnes mises en cause, ou de leurs proches (victimes d'agression, accident ou de suicide), est particulièrement délicate, voire épineuse pour les professionnels de l'information. Doit-on nommer ou ne pas nommer? La règle qui s'impose, eu égard à cette question, est identique à celle déjà énoncée pour le traitement de ces affaires : ne révéler l'identité des personnes que lorsque cette identification est d'un intérêt public certain, voire incontournable. " (Conseil de presse du Québec, 2003, p. 29-30)

Concernant les drames humains, l'article $18.1 \mathrm{du}$ code du CPQ précise également qu'il est reconnu par la déontologie que : "Les journalistes et les médias d'information font preuve de retenue et de respect à l'égard des personnes qui viennent de vivre un drame humain et de leurs proches. Ils évitent de les harceler pour obtenir de l'information et respectent leur refus d'accorder une entrevue " (CPQ, 2015a, p. 26).

Cependant, en scrutant les réseaux sociaux utilisés par les responsables des attaques analysées, dans le but d'en rapporter des détails importants et en s’intéressant de près aux parents et aux membres de la famille des auteurs de ces attaques ainsi que des victimes, les médias ont possiblement atteint les limites d'application des principes concernant l'identification des proches et le respect du refus d'accorder des entrevues. Il est donc légitime de soulever un questionnement à savoir si les médias ont outrepassé la frontière de ce qui peut être qualifié d'intérêt public en s'intéressant de près aux familles des victimes et en révélant des détails qui auraient pu compromettre leur vie privée, voir même leur sécurité.

Ainsi, après les événements de Saint-Jean-surRichelieu, les médias ont recueilli les témoignages de voisins et d'amis de Couture-Rouleau. Ils se sont intéressés à sa vie sociale, à sa vie de famille avec son ex-conjointe et son enfant, ainsi qu'à sa vie professionnelle. Ils ont surtout largement questionné son père, Gilles Rouleau, d'autant plus que ce dernier s'inquiétait de la conversion de son fils et avait alerté les autorités à plusieurs reprises à ce propos.

Au lendemain des événements de Saint-Jean-surRichelieu, Gilles Rouleau déclarait aux médias : «J'ai perdu mon fils. Mon fils est partout ce matin. Laissez-moi tranquille, je n'ai aucun commentaire. " (Société Radio-Canada, 21 octobre 2014), propos qui ont été relayés sur diverses tribunes publiques. Or, 
devant une certaine pression médiatique, Rouleau a finalement dû se prononcer publiquement et son image a été largement diffusée. Il a alors notamment appelé à un renforcement du pouvoir des services de sécurité canadiens et a déploré l'impuissance des autorités à stopper son fils avant le drame. La détresse émotionnelle de Gilles Rouleau est encore palpable dans divers propos qu'il a tenus en entrevues dont celui-ci : " "J'ai mal. Je dois vivre avec ce qu’il a laissé, je suis en 'câlisse" après lui", a-t-il ajouté, la gorge nouée par l'émotion. Gilles Rouleau dit coopérer pleinement avec l'enquête en cours et avoir donné aux policiers un plein accès à sa résidence, où son fils habitait le sous-sol " (Alteresco, 24 octobre 2014). Dans ce même article, on invite M. Rouleau à commenter la fusillade d'Ottawa, bien qu'il n'y ait pas de lien établi avec les actes commis par son fils, on lui demande aussi de se prononcer sur le problème de radicalisation des jeunes Canadiens.

L'exemple encore plus frappant du difficile équilibre à trouver pour les médias entre respect de la personne et de la vie privée et information d'intérêt public s'est sans doute révélé dans les heures qui ont suivi l'attentat survenu sur la colline parlementaire d'Ottawa. Les médias ont alors rapidement cherché à en apprendre le plus possible sur l'auteur de la fusillade Michael Zehaf-Bibeau. Ils ont rapidement révélé qu’il était né à Montréal, qu’il avait grandi au Québec avant de déménager dans l'Ouest canadien, mais surtout, ils ont révélé l'identité de ses parents, dont celle de sa mère Susan Bibeau, dévoilant du même coup qu'elle était une haute fonctionnaire fédérale en poste comme vice-présidente de la Section de l'immigration de la Commission de l'immigration et du statut de réfugié du Canada. Ces informations ont rendu cette dernière très facile à identifier et auraient sans doute pu lui occasionner des complications au travail. Certains médias ont même souligné " l'ironie " de la situation, dressant un parallèle entre le poste occupé par Mme Bibeau et les actes commis par son fils. Ils ont également dévoilé l'identité du père de Zehaf-Bibeau, Bulgasem Zehaf, précisant qu'il était immigré, d'origine libyenne, et qu'il était retourné en de nombreuses occasions au pays. On peut également soulever ici la question à savoir s'il était d’intérêt public, mais également s'il pouvait s'avérer discriminatoire, de mentionner l'origine du père de Zehaf-Bibeau. À ce propos, le CPQ mentionne qu'il «n'est pas interdit aux médias de faire état des caractéristiques qui différencient les personnes ou les groupes. Cependant, cette mention doit être pertinente et d'intérêt public, ou être une condition essentielle à la compréhension et à la cohérence de l'information " (Conseil de presse du Québec, 2003, p. 41). Certes, l'origine du père n'était pas ici une condition essentielle à la compréhension des faits, mais pouvait insinuer un lien entre l'origine de ce dernier et les allégeances du fils qui s'était radicalisé, ce qui peut avoir pour effet d'engendrer des préjugés au sein de la population.

Après avoir été ainsi identifiée et avoir vu sa photo publiée dans les médias, Susan Bibeau a décidé elle aussi de réagir publiquement, ce qu'elle a fait dans une lettre qu'elle a fait parvenir aux médias, où elle soutient être " horrifiée " et "fâchée " par les gestes que son fils a commis. Elle tente de les comprendre et de les expliquer en revenant notamment sur le refus des autorités canadiennes de délivrer un passeport à ce dernier pour se rendre en Arabie Saoudite ainsi qu'en évoquant sa détresse et son désespoir. Elle mentionne son propre sentiment de culpabilité et s'excuse publiquement. Il est difficile de trouver, dans un cas comme celui-ci, où se situe le juste équilibre entre le respect de la peine et de la vie privée des parents, qui sont eux aussi des victimes de ces événements, et le devoir des médias de comprendre la situation et de tenter d'expliquer les motivations du tireur. Ainsi, à l'intérieur du corpus que nous avons analysé, une journaliste du Journal de Montréal mentionne que puisque "les réflexions et les observations de la mère de Michael Zehaf-Bibeau apportent un éclairage de première source quant aux possibles motivations derrière les événements tragiques d'Ottawa ", il lui semble pertinent de les diffuser (Legault, 25 octobre 2014).

Bien que l'intérêt pour les propos de Susan Bibeau puisse se justifier, selon certains médias, par le besoin de mieux comprendre les motivations du tireur et de déterminer s'il s'agit d'un acte terroriste ou non, certains autres éléments largement couverts semblent plus facilement assimilables à la curiosité publique. C'est le cas de l'intérêt que les médias ont porté à la résidence familiale de Susan Bibeau et de Bulgasem Zehaf, où ne résidait plus Michael Zehaf-Bibeau. Plusieurs médias ont tourné des reportages et pris des photos devant cette dernière, espérant pouvoir interroger des gens qui entraient ou sortaient des lieux. Certains médias ont même ouvertement diffusé le nom de la rue sur laquelle se situe la maison et ont mentionné le nom de son quartier, rendant celle-ci facilement localisable, ce qui aurait pu mettre en danger ses occupants. Une photo d'Olivier Jean diffusée le 23 octobre 2014 dans le quotidien La Presse et prise par une fenêtre extérieure permet même de voir l'intérieur de la maison, dans le but de démontrer que les parents devaient eux aussi faire face aux images de l'attentat au Parlement d'Ottawa et regardaient les nouvelles télévisées qui traitaient du drame. Un autre exemple tiré de notre corpus qui porte à réflexion est celui d'un duplex entre le présentateur Pierre Bruneau et la journaliste Marie-Laurence Delainey, publié le 23 octobre 2014 sur le site de TVA Nouvelles, tour- 
né en direct devant la maison de Mme Bibeau, qui mentionne une présence policière afin de réduire le nombre de médias sur les lieux, alors que la journaliste poursuit son intervention devant le domicile, où l'on voit également l'adresse civique ainsi que des images de l'intérieur du domicile, telles des photos des parents, tournées encore une fois à travers les fenêtres.

Dans un autre article paru dans le quotidien $\mathrm{La}$ Presse du 24 octobre 2014, la tante de Zehaf-Bibeau est à son tour interrogée, après qu'on ait appris que ce dernier s'était présenté au chalet de sa tante, situé à Mont-Tremblant au Québec, la veille des événements d'Ottawa, pour y souper et y coucher. Dans cet article, encore une fois, le chalet de Monique Bibeau est clairement illustré dans une image d'Olivier Pontbriand, où l'on aperçoit également les voitures et les plaques d'immatriculation.

Au terme de ces observations et à la suite de la diffusion des images relevées lors de l'analyse, il est certes possible de se demander si les médias ont outrepassé l'intérêt public pour assouvir une certaine curiosité liée au désir de mieux comprendre le quotidien et les motivations de Zehaf-Bibeau et de Couture-Rouleau. Dans une volonté, certes noble, d'expliquer les événements et de s'interroger sur le phénomène de la radicalisation ainsi que d'évaluer le niveau de sécurité des Canadiens et de mieux comprendre le terrorisme, les médias sont-ils allés trop loin dans la couverture des événements d'octobre 2014 ?

Bien que certains médias internationaux aient, en partie, salué la qualité du travail réalisé par les médias canadiens lors de la difficile couverture de ces événements, alors que les sources d'informations étaient nombreuses et que les impératifs particuliers du direct se faisaient sentir, les réflexions éthiques et déontologiques que notre analyse de la couverture médiatique québécoise des événements nous amène à soulever démontrent, encore une fois, les difficultés liées à la couverture journalistique des situations de crises, en particulier lors d'événements dramatiques de grande ampleur pouvant être assimilés à des actes terroristes.

Lors d'une recherche précédente portant sur la couverture de crise, incluant la guerre et le terrorisme, nous soulevions que les professionnels de l'information " tentent de rapporter les faits au meilleur de leur connaissance, tout en devant départager l'information d'intérêt public de la curiosité publique qui s'installe et qui pousse parfois certains journalistes à s'acharner sur les victimes ou sur leur famille pour susciter de l'émotion " (Carignan, 2014, p. 270), c'est ce que nous observons encore une fois avec les événements d’octobre 2014. Ces événements confirment également la conclusion de Bettez qui estimait que les caractéristiques du journalisme en situation d'exception étaient "difficilement conciliables avec plusieurs fondements juridiques et philosophiques de la déontologie journalistique " (Bettez, 2010, p. 71). Le travail pour concilier ces deux aspects n’est certes pas terminé.

\section{IMPLICATIONS ET CONCLUSION}

La théorie de la responsabilité sociale des médias, qui est au cœur du principe d'autorégulation médiatique, suppose une liberté imputable des médias qui doivent s'assurer de répondre aux besoins du public. Elle soutient que "les médias ont une trop grande influence sur la marche de la société pour qu'ils puissent prendre leur rôle à la légère " (Bernard, 2005, p. 46). Or, plusieurs auteurs critiquent la méconnaissance de cette théorie et son inefficacité, affirmant qu'elle "représente l'idéologie officieuse des médias tout en demeurant un modèle, un objectif aux contours vagues, largement ignoré dans la pratique » (Saint-Jean, 2002, p. 93). Lorsque nous nous intéressons aux limites de fonctionnement actuelles des instances d'autorégulation de la presse, telles de Conseil de presse du Québec, qui vit à répétition des crises financières et structurelles mettant en danger sa survie, qui essuie de la part de médias et des publics des critiques sur la rigueur de ses décisions et sur leur impact limité et qui s'autosaisie peu de cas présentant des problèmes majeurs pour la profession journalistique, repensons ici au comité avorté sur l'étude du traitement des crises, il y a lieu de s'interroger sur les limites actuelles de la théorie de la responsabilité sociale de la presse.

L'analyse de la couverture médiatique des événements d'octobre 2014 met en lumière la difficulté pour les médias et les professionnels de l'information d'appliquer des principes déontologiques très généraux et parfois peu étoffés lors de la couverture de sujets sensibles, dans un contexte de traitement accéléré de l'information. Il faut parfois aller fouiller en profondeur dans la jurisprudence de l'organisme pour trouver des explications et des exemples d'application pouvant aider à mieux définir ces principes et à les appliquer à la couverture d'attaques armées comme celles d'Ottawa et de Saint-Jean-surRichelieu. Il est certain que, dans le feu de l'action, les professionnels de l'information recherchent des outils pouvant aider rapidement aux prises de décisions et ne peuvent se permettre une telle recherche pour nourrir leur réflexion. Il semble donc nécessaire de créer des outils déontologiques mieux adaptés aux nouvelles réalités de la presse et l'aidant à respecter les principes fondateurs de l'éthique et de 
la déontologie. D’ailleurs, notre analyse nous permet de constater l'absence, dans la dernière édition du guide déontologique du CPQ, de certains principes déontologiques pertinents et couramment soulevés dans la jurisprudence de l'organisme. Une analyse de contenu des différentes versions de ce guide et une comparaison avec les autres guides d'organismes d'autorégulation de la presse nous sembleraient pertinentes pour apporter un éclairage plus approfondi sur ce point ${ }^{8}$. Cette étude met également en lumière le besoin de préciser les contours de certaines notions largement utilisées par la profes- sion journalistique et les organismes d'autorégulation, mais qui ne sont pas définies de façon précise, pensons notamment à l'intérêt public, à la curiosité publique ou à l'indépendance journalistique. Des expressions largement répandues sans qu'on analyse leur véritable sens.

Date de soumission de l'article : 15 avril 2016. Date d'acceptation : 31 octobre 2016.

\section{Notes}

1. La non-reconnaissance de la légitimité du CPQ et le refus systématique de la part de Québecor de répondre aux plaintes le concernant affectent le fonctionnement de l'organisme alors que le groupe détient $40 \%$ de l'information totale produite au Québec selon la Fédération professionnelle des journalistes du Québec (2014). Depuis ce départ, d'autres entreprises de presse ont aussi épisodiquement menacé de quitter le CPQ, ce que rapportent également Corriveau et Sirois (2012).

2. Le manque de diffusion des décisions du CPQ est notamment soulevé par Bernier (2010). Il figure également parmi les conclusions du rapport L'état de la situation médiatique au Québec : l'avis du public, relatant les conclusions de la tournée des régions du Québec effectuée par le CPQ en 2008 : "Il existe un préjugé voulant que déposer une plainte devant le Conseil de presse n'ait aucune conséquence. Le public estime que ces décisions ne sont pas assez diffusées dans la sphère publique et médiatique, notamment lorsque le média est concerné » (CPQ, 2008, 25)

3. «État Islamique » est le nom officiel sous lequel est inscrit ce groupe militant sunnite sur la liste des entités terroristes inscrites par Sécurité publique Canada (2016). C'est pourquoi nous utiliserons cette appellation à l'intérieur de cet article, bien que cette entité soit connue sous différents noms dont « al-Qaïda en Irak », "État islamique en Irak et au Levant » (EIIL), «État islamique en Irak et en Syrie » (EIIS) ou « État islamique d’Irak et d'al-Cham » (EIIC)

4. La communauté internationale a du mal à s'entendre sur la définition du terrorisme, dont le terme a une forte connotation négative, puisqu'il " constitue un moyen au service d'une fin. De ce fait, sa nature varie considérablement selon les cas. Seul le principe général, purement utilitaire, et certains mécanismes de l'action favorisent une représentation unitaire du phénomène " (Larousse, 2015), ce qui pousse plusieurs chercheurs à inviter à la prudence pour en faire usage.
5. Le jour même de l'attaque de Saint-Jean-sur-Richelieu, le ministre fédéral conservateur, Steven Blainey, a associé cet événement à un acte terroriste, affirmant qu'il démontrait que la menace terroriste est "bien réelle " au Canada (Marquis, 21 octobre 2014). Par ailleurs, dans une adresse à la nation présentée le soir même des événements d'Ottawa, l'ex premier ministre canadien Stephen Harper parlait déjà d'un acte terroriste, précisant que le " Canada ne sera jamais intimidé » et qu'il renforcerait ses mesures de sécurité. Harper ajoutait également que, dans les « prochains jours, nous en apprendrons davantage sur le terroriste et ses complices, mais les événements de cette semaine nous rappellent tristement que le Canada n'est pas à l'abri des attaques - des types d'attaques que nous avons vues ailleurs dans le monde".

6. Un album souvenir réalisé pour les élèves de terminale au Canada.

7. « Câlisse » est un juron couramment utilisé dans le langage québécois et inspiré des termes sacrés de la religion catholique. Il sert ici à exprimer des émotions, telles la colère et la frustration.

8. Exercice déjà entamé par Raymond Corriveau dans La déontologie du Conseil de presse du Québec, une mutation bénéfique?, Actes des Travaux du Comité de Recherche "Sociologie de la communication ", Congrès AISLF, Montréal, 5 au 7 juillet 2016, disponibles au https://web.univ-pau.fr/RECHERCHE/SET/ AISLFCR33/DOCS_SOCIO/2016/Actes_AISLF_CR33_2016_Montreal.pdf 


\section{RÉFÉRENCES BIBLIOGRAPHIQUES}

Alteresco, T., 2014, 24 oct., « Attentat à Saint-Jean-surRichelieu : "La loi doit changer", dit le père de Martin Couture-Rouleau ", Société Radio-Canada, http://ici.radiocanada.ca/nouvelles/societe/2014/10/24/005-pere-couturerouleau-temoignage-saint-jean-sur-richelieu.shtml.

Beck, U., 2001, La société du risque. Sur la voie d'une autre modernité, Paris, Flammarion.

Bernard, J., 2005, La théorie de la responsabilité sociale de la presse : Présentation, discussion, actualisation, Mémoire de maîtrise, Université de Sherbrooke, http:// savoirs.usherbrooke.ca/handle/11143/5262.

Bernier, M.-F., 2013, « La montée en puissance d'un "5e pouvoir": Les citoyens comme acteurs de la corégulation des médias ? », Éthique publique, vol. 15, no 1, pp. 169-191.

Bernier, M.-F., 2010, «Fin du mythe de l'autorégulation des médias ", in Fahmy, M. (Éd.), L'état du Québec 2010, Louiseville, Les Éditions du Boréal.

Berthomet, S., 2015, La Fabrique du djihad : Radicalisation et terrorisme au Canada, Montréal, EDITO.

Bettez, G., 2010, La déontologie et pratiques journalistiques lors de situations d'exception : Le cas de la fusillade au collège Dawson, Essai de maîtrise inédit, Université du Québec Trois-Rivières.

Boutté, G., 2006, Risques et catastrophes : Comment éviter et prévenir les crises? Paris, Éditions du Papyrus.

Brillon, P., 2007, 19 avr., «Un peu de retenue S.V.P. », La Presse.

Burton, F., Stewart, S., 2008, The 'Lone Wolf’ Disconnect, STRATFOR Global Intelligence, http://www.stratfor.com/ weekly/lone_wolf_disconnect.

Carey, J. W., 1986, « The Dark Continent of American Journalism », in Manoff, R. K., Schudson, M. (Éds.), Reading the News, New York, Pantheon, pp. 146-196.

Carignan, M.-E., 2014, La modification des pratiques journalistiques et du contenu des nouvelles télévisées, du quotidien à la situation de crise : Analyse France/Québec, Thèse de doctorat inédite, Université de Montréal, Sciences-po Aix-en-Provence.

Centers for Disease Control and Prevention, 2012, Crisis and Emergency Risk Communication, http://emergency. cdc.gov/cerc/resources/pdf/cerc_2012edition.pdf.

Champagne, P., 1999, « La médiatisation des risques et l'espace public », Actes de la 13e séance du séminaire du Programme risques collectifs et situations de crise, Paris, CNRS. Code criminel, R.C.S., 1985, c. C-46, art. 83.01.

Collard, N., 2014, 24 oct., « Le style de Peter Mansbridge impressionne les Américains ", La Presse, http://www. lapresse.ca/arts/medias/201410/24/01-4812168-le-style-depeter-mansbridge-impressionne-les-americains.php.

Conseil de presse du Québec, 2016, Le Conseil, http:// conseildepresse.qc.ca/le-conseil/mission/.

Conseil de presse du Québec, 2015a, Guide de déontologie journalistique du Conseil de presse du Québec, http:// conseildepresse.qc.ca/wp-content/uploads/2015/08/Guidede-d\%C3\%A9ontologie-journalistique_CPQ.pdf
Conseil de presse du Québec, 2015b, Rapport d'activités 2014-2015, http://conseildepresse.qc.ca/wp-content/ uploads/2016/02/rapport-dactivit\%C3\%A9s-2014-2015.pdf. Conseil de presse du Québec, 2008, L'état de la situation médiatique au Québec : L'avis du public, Montréal, http://conseildepresse.qc.ca/wp-content/ uploads/2013/01/2008-11-10_etat-situation-mediatique_ avis-du-public.pdf.

Conseil de presse du Québec, 2004, Décision D2004-05059, http://conseildepresse.qc.ca/decisions/d2004-05-059/.

Conseil de presse du Québec, 2003, Droits et responsabilités de la presse (3e éd.), Montréal.

Corriveau, R., Sirois, G., 2012, L'information : La nécessaire perspective citoyenne, Québec, Presses de l'Université du Québec.

Deland, M., 2014, 20 oct., « Deux militaires happés par un chauffard : Le suspect a dit "agir au nom d'Allah" ", Agence QMI, TVA Nouvelles, http://tvanouvelles.ca/lcn/ infos/faitsdivers/archives/2014/10/20141020-154216.html.

Fédération professionnelle des journalistes du Québec, 2014, Candidature de Pierre Karl Péladeau : Le mélange de l'État et de la presse est explosif, http://www.fpjq.org/ candidature-de-pierre-karl-peladeau-le-melange-de-letat-etde-la-presse-est-explosif-3/.

Fengler, S., Eberwein, T., Alsius, S., Baisnée, O. Bichler, K., Dobek-Ostrowska, B., Powell, W., 2015, « How Effective is Media Self-Regulation ? Results from a Comparative Survey of European Journalists ", European Journal of Communication, vol. 30, no 3, pp. 249-266.

Fischhoff, B., Slovic, P., Lichtenstein, S., Read, S., Combs, B., 1978, "How Safe is Safe Enough ? A Psychometric Study of Attitudes towards Technological Risks and Benefits, Policy Sciences, vol. 9, no 2, pp. 127-152.

Giddens, A., 2005, La constitution de la société : Éléments de la théorie de la structuration, Paris, Presses universitaires de France.

Giddens, A., 2000, Les conséquences de la modernité, Paris, L'Harmattan.

Gollom, M., 2014, 27 oct., « Michael Zehaf-Bibeau and Martin Couture-Rouleau : Their Shared Traits ", CBC News, http://www.cbc.ca/news/canada/michael-zehaf-bibeau-andmartin-couture-rouleau-their-shared-traits-1.2812241.

Habermas, J., 1991, L'espace public. Archéologie de la publicité comme dimension constitutive de la société bourgeoise, Paris, Payot.

Hénin, N., 2015, Jihad academy : Nos erreurs face à l'état islamique, Paris, Fayard.

La Presse, 2014, 20 oct., "Acte terroriste à Saint-Jean : Martin "Ahmad" Rouleau inspiré par l'islamisme radical ", La Presse, http://www.lapresse.ca/actualites/dossiers/ attentat-a-st-jean-sur-richelieu/201410/20/01-4810905-acteterroriste-a-saint-jean-martin-ahmad-rouleau-inspire-parlislamisme-radical.php.

Lagacé, P., 2014, 6 juin, "Un tueur, une photo », La Presse, http://www.lapresse.ca/debats/chroniques/patricklagace/201406/06/01-4773342-un-tueur-une-photo.php. 
Lagadec, P., 2008, 14 nov., La grande décision : Capitulation ou invention, face aux événements extrêmes, http:// www.patricklagadec.net/fr/pdf/La_grande_decision.pdf.

Le Monde et AFP, 2014, 23 oct., «Attentat à Ottawa : Le tireur espérait partir pour la Syrie », Le Monde, http:// www.lemonde.fr/ameriques/article/2014/10/23/le-canada-traumatise-apres-l-attaque-du-parlement-par-un-tireurisole_4510844_3222.html.

Legault, J., 2014, «Fusillade d'Ottawa : Réflexions de la mère de Zehaf-Bibeau ", Le Journal de Montréal, http:// www.journaldemontreal.com/2014/10/25/fusillade-dottawa-reflexions-de-la-mere-de-zehaf-bibeau.

Leveson, B., 2012, nov., An Inquiry into the Culture, Practices and Ethics of the Press, Volume 1, Londres, The Stationery Office, https://www.gov.uk/government/uploads system/uploads/attachment_data/file/270939/0780_i.pdf.

Magos, V., 2014, 15 sept., «Il faut refuser de regarder les images des mises à mort ", Slate.fr, http://www.slate.fr/ story/92145/refuser-regarder-images-etat-islamique.

Marquis, M., 2014, 21 oct., « Attentat de St-Jean : un acte “ terroriste” ? ", La Presse, http://www.lapresse.ca/actualites/dossiers/attentat-a-st-jean-sur-richelieu/201410/21/014811177-attentat-de-st-jean-un-acte-terroriste.php.

Miège, B. et al., 1986, Le JT : Mise en scène de l'actualité à la télévision, Paris, La Documentation française.

Nacos, B. L., 2005, Les médias et le terrorisme, Montréal, Éditions Saint-Martin.

Pêcheux, M., 1988, "Discourse : Structure or Event?", in Nelson, C., Grossberg, L. (Éds.), Marxism and Interpretation of Culture, Urbana, University of Illinois Press, pp. 633-650.

Peretti-Watel, P., 2001, La société du risque, Paris, La Découverte.

Peritz, I., Thanh Ha, T., Perreaux, L., 2014, 21 oct., «Martin Couture-Rouleau's Shift into Extremism Played Out on Social Media », The Globe and Mail, http://www. theglobeandmail.com/news/national/extremism-in-canadas-borders/article21217185/.

Raboy, M., 1993, Crise des médias, crises de société : Les femmes, les hommes et l'École polytechnique de Montréal, Communication : information, médias, théories, pratiques, vol. 14, no 1, p. 82.

Radio-Canada avec La Presse Canadienne, 2014, 8 déc., "Vidéo de djihadistes : La SQ presse les médias d'agir avec discernement ", Société Radio-Canada, http://ici. radio-canada.ca/nouvelles/national/2014/12/08/002-conseilnational-musulmans-john-maguire-video-terrorisme-etat-islamique-canada.shtml.

Ricard-Châtelain, B., 2014, 22 oct. « Les militaires sommés de laisser leur uniforme à la maison », Le Soleil, http://www. lapresse.ca/le-soleil/actualites/politique/201410/22/014811623-les-militaires-sommes-de-laisser-leur-uniforme-ala-maison.php.
Saint-Jean, A., 2002, Éthique de l'information : Fondements et pratiques au Québec depuis 1960, Montréal, Presses de l'Université de Montréal.

Sécurité publique Canada, 2016, Lutte contre le terrorisme, http://www.securitepublique.gc.ca/cnt/ntnl-scrt/ cntr-trrrsm/index-fr.aspx.

Siebert, F. S., Peterson, T., Schramm, W., 1956, Four Theories of the Press : The Authoritarian, Libertarian, and Soviet Communist Concepts of What Press Should Be and Do, Champaign, University of Illinois Press.

Sioui, M.-M., 2014, 24 oct., " Martin Couture-Rouleau : "il avait le coeur noble et le cerveau déprimé" ", La Presse, http://www.lapresse.ca/actualites/dossiers/ attentat-a-st-jean-sur-richelieu/201410/23/01-4812127-martin-couture-rouleau-il-avait-le-coeur-noble-et-le-cerveau-deprime.php.

Société Radio-Canada, 2015, 6 mars, "Zehaf-Bibeau voulait "venger les moudjahidines du monde entier" ", Société Radio-Canada, http://ici.radio-canada.ca/nouvelles/ politique/2015/03/06/001-devoilement-video-zehaf-bibeau. shtml.

Société Radio-Canada, 2014, 9 déc., «Attentat à SaintJean-sur-Richelieu », Société Radio-Canada, http://ici. radio-canada.ca/sujet/attentat-saint-jean-sur-richelieu.

Société Radio-Canada, 2014, 23 oct., "Fusillade au parlement d'Ottawa : Qui était Michael Zehaf-Bibeau, le tireur au parlement ? ", Société Radio-Canada, http://ici. radio-canada.ca/nouvelles/societe/2014/10/22/015-famillemichael-zehaf-bibeau-passe.shtml.

Société Radio-Canada, 2014, 21 oct., "Attentat à SaintJean-sur-Richelieu : Martin "Ahmad” Couture-Rouleau, un adepte de l'islam radical ", Société Radio-Canada, http:// ici.radio-canada.ca/nouvelles/societe/2014/10/21/003-attentat-saint-jean-sur-richelieu-martin-rouleau-portrait.shtml.

Spaaij, R., 2012, Understanding Lone Wolf Terrorism : Global Patterns, Motivations and Prevention, Dordrecht, Springer Netherlands.

Terrorisme, (s.d.), Larousse Encyclopédie, http://www.larousse.fr/encyclopedie/divers/terrorisme/96706, consulté le $15 / 04 / 15$.

Tuchman, G., 1978, Making News : A Study in the Construction of Reality, New York, The Free Press.

Tsfati, Y., Weimann, G., 2002, « www.terrorism. com: Terror on the Internet », Studies in Conflict \& Terrorism, vol. 25, no 5, pp. 317-332.

West, J., 2014, « Canada's Coverage of the Ottawa Shootings Put American Cable News to Shame », Mother Jones, http://www.motherjones.com/mixed-media/2014/10/cbc-ottawa-shootings-cable-news. 


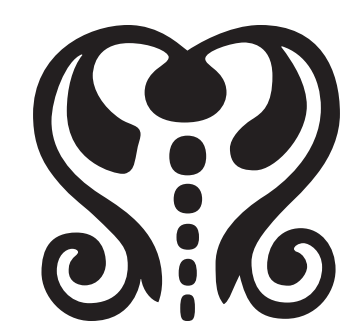




\section{Résumé | Abstract | Resumo}

Quelle responsabilité sociale de la presse?

La couverture des attaques à Ottawa et à Saint-Jean-sur-Richelieu

What social responsibility for the press?

The coverage of the attacks in Ottawa and Saint-Jean-sur-Richelieu

Qual a responsabilidade social da imprensa?

A cobertura dos ataques a Otava e Saint-Jean-sur-Richelieu

Fr.

Dans cet article, l'auteure s'intéresse au contexte entourant la création des instances d'autorégulation médiatique en revenant sur les fondements de la théorie guides déontologiques d'organismes d'encadrement des médias, dont le Conseil de presse du Québec. L'évolution des pratiques professionnelles journalistiques amène un questionnement sur les limites actuelles de ce type d'encadrement pour couvrir les situations de crise, alors que la société, qualifiée de « société du risque " par plusieurs auteurs, est confrontée à un nombre croissant de dangers et que le cadre de pratique amène les journalistes à couvrir davantage ce type d'événement. S'intéressant en particulier au cas des attaques survenues à Ottawa et à Saint-Jean-sur-Richelieu au Canada en octobre 2014, l'auteure met en parallèle différentes pratiques observées chez les médias canadiens avec les principaux griefs relevés dans la jurisprudence du Conseil de presse du Québec et les énoncés déontologiques du Guide de déontologie journalistique du Conseil de presse du Québec, dans le but de tracer les limites potentielles d'application des principes de l'organisme en contexte critique. Cette analyse permettra d'entamer une réflexion sur la viabilité de la théorie de la responsabilité sociale de la presse et des processus d'autorégulation médiatiques actuellement en place ainsi que sur le rapport entre liberté de presse et droits individuels (vie privée, sécurité, respect des personnes) de même qu'entre intérêt public et curiosité publique.

Mots-clés :déontologie, attentats, risque, médias, théorie de la responsabilité sociale de la presse

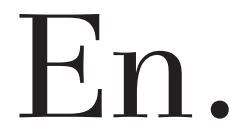

This article examines the context surrounding the creation of self-regulation entities in the media sector by analyzing the origins of the social responsibility of the press theory. This theory is used as a guideline to establish the deontological principles of self-regulation organizations such as the Quebec Press Council. The evolution of professional journalistic practices bring a questioning about the current limitations of this type of supervision to cover crisis, while society, described as a "risk society" by several authors, is facing a growing number of dangers and while the professional journalistic context brings the journalists to cover even more this type of event. By focusing on the case of the October 2014 attacks in Ottawa and in Saint-Jean-sur-Richelieu, Canada, the author observes various Canadian media practices and studies them in parallel with the main objections raised in the Quebec Press Council jurisprudence and with the ethic guideline of this organization, to observe the potential limitations of these principles in a context of crisis. This analysis will lead to a reflection on the viability of the social responsibility of the press theory and on the self-regulation process currently in place. It will also question the relation between freedom of the press and individual rights (attitude of the press towards individuals and groups, privacy, security) as well as between public interest and public curiosity.

Keywords: deontology, risk, terrorism, media, social responsibility of the press 
Pt.

Este artigo é discute o contexto de criação de instâncias de auto-regulação mediática, por meio de uma análise dos fundamentos da teoria da responsabideontológicas dos organismos de enquadramento dos media, nomeadamente o Conselho de imprensa do Quebeque. A evolução das práticas profissionais jornalísticas originou um questionamento sobre os limites actuais desse tipo de enquadramento para cobrir situações de crise, enquantoa sociedade, descrita como "sociedade de risco" por diversos autores, é confrontada com um número crescente de perigos e o quadro da prática leva os jornalistas a dar mais importância à cobertura desse tipo de eventos. Com um interesse particular sobre o caso dos atentados ocorridos em Otava e Saint-Jean-sur-Richelieu em Outubro de 2014, a autora coloca em paralelo diferentes práticas observadas nos media canadianos com as principais objecções identificadas na jurisprudência do Conselho de imprensa do Quebeque e os enunciados deontológicos do Guia de deontologia jornalística do Conselho de imprensa do Quebeque, com o objectivo de observar os potenciais limites da aplicação dos princípios do organismo em contexto crítico. Esta análise permite levar a cabo uma reflexão sobre a viabilidade da teoria da responsabilidade social da imprensa e os processos de auto-regulação mediáticos actualmente em vigor bem como sobre a relação entre liberdade de imprensa e direitos individuais (privacidade, segurança, respeito das pessoas) e a relação entre interesse público e curiosidade pública.

Palavras-chave: deontologia, crise, terrorismo, media, teoria da responsabilidade social da imprensa

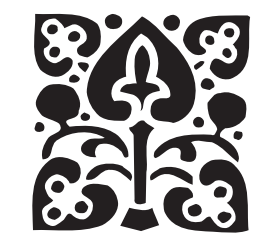

